\title{
Polymicrobial and Monomicrobial Infections after Spinal Surgery: A Retrospective Study to Determine which Infection is more Severe
}

\author{
Shaoqiang Liu ${ }^{1,2}$, Qiang $\mathrm{Qi}^{1}$, Zhongqiang $\mathrm{Chen}^{1}$, Ning $\mathrm{Liu}^{1}$, Zhaoqing Guo ${ }^{1}$, \\ Chuiguo Sun ${ }^{1}$, Weishi $\mathrm{Li}^{1}$, Yan Zeng ${ }^{1}$, Zhongjun Liu ${ }^{1}$ \\ ${ }^{1}$ Department of Orthopedics, Peking University Third Hospital, Beijing, China \\ ${ }^{2}$ Department of Spine Surgery, Fuzhou Second Hospital of Xiamen University, Fuzhou, China
}

\section{Study Design: A retrospective clinical review.}

Purpose: To investigate the difference in clinical manifestations and severity between polymicrobial and monomicrobial infections after spinal surgery.

Overview of Literature: Surgical site infections (SSIs) after spinal surgery are a major diagnostic and therapeutic challenge for spinal surgeons. Polymicrobial infections after spinal surgery seem to result in poorer outcomes than monomicrobial infections because of complementary resistance to antibiotics. However, comparison of the clinical manifestations and severity between polymicrobial and monomicrobial infections are limited.

Methods: Sixty-seven patients with SSIs after spinal surgery were studied: 20 patients with polymicrobial infections and 47 with monomicrobial infections. Pathogenic bacteria identified were counted and classified. Age, sex, and body mass index were compared between the two groups to identify homogeneity. The groups were compared for clinical manifestations by surgical site, postoperative time to infection, infection site, incisional drainage, incisional swelling, incisional pain, neurological signs, temperature, white blood cell count, and the percentage of neutrophils. Finally, the groups were compared for severity by hospital stay, number of rehospitalizations, number of debridements, duration of antibiotics administration, number of antibiotics administered, and implant removal.

Results: Polymicrobial infections comprised $29.9 \%$ of SSIs after spinal surgery, and most polymicrobial infections (70.0\%) were caused by two species of bacteria only. There was no difference between the groups in terms of clinical manifestations and severity. In total, 96 bacterial strains were isolated from the spinal wounds: 60 strains were gram-positive and 36 were gram-negative pathogenic bacteria. Staphylococcus aureus, Staphylococcus epidermidis, Escherichia coli, and Enterobacter cloacae were cultured in order of the frequency of appearance.

Conclusions: Most polymicrobial infections were caused by two bacterial species after spinal surgery. There was no difference in clinical manifestations or severity between polymicrobial and monomicrobial infections.

Keywords: Spine; Postoperative complications; Surgical wound infection; Coinfecion

Received Aug10, 2016; Revised Dec 12, 2016; Accepted Dec 18, 2016

Corresponding author: Qiang Qi

Department of Orthopedics, Peking University Third Hospital,

No. 49 Huayuanbei Rd, Haidian District, Beijing 100191, China

Tel: +86-010-82267388, Fax: +86-010-82267388, E-mail: qiqiang_puth@sohu.com 


\section{Introduction}

Spinal infections are a major diagnostic and therapeutic challenge for spinal surgeons. Surgical site infections (SSIs) after spinal surgery, which occur at a rate of $0.7 \%-12.2 \%$ [1-4], typically result in higher postoperative morbidity, mortality, and health care costs. In particular, health care costs can increase by as much as four-fold [5] because of the necessary prolonged hospitalization, higher dosage of antibiotics, multiple debridements, or even removal of the implants [6].

An SSI can be due to either a single microbial species (monomicrobial) or multiple species (polymicrobial). Proof of polymicrobial infection is generally based on the isolation of $\geq 2$ species of microorganisms from $\geq 2$ cultures of secretion from the incision or intraoperative tissue specimens; or the isolation of $\geq 2$ species in $\geq 1$ intraoperative cultures and evidence of infection in the surgical site (e.g., purulence, acute inflammation, or a sinus tract communicating with the surgical site) $[7,8]$. The involvement of multiple species makes SSIs relatively more difficult to control and treat.

After spinal surgery, polymicrobial SSIs seem to be associated with higher rates of treatment failure, often due to complementary cross-species resistance to a diverse set of antibiotics [9]. However, comparisons of the clinical manifestations and severity between polymicrobial and monomicrobial infections in previous investigations are limited. This study was designed to investigate the differences in clinical manifestations and severity between polymicrobial and monomicrobial infections after spinal surgery.

\section{Materials and Methods}

\section{Patients}

Between July 2004 and December 2012, a total of 28,778 patients underwent spinal surgery. All the patients were administrated cefuroxime (Zinacef, GlaxoSmithKline, Verona, Italy) prophylactic antibiotics 30 minutes before the skin incision and preferably for 48 hours as an intravenous infusion postoperatively.

In total, 108 patients were identified with SSIs after spinal surgery, and the total infection rate was $0.38 \%$. Among them, 41 patients with SSIs (38.0\%) with culturenegative results were excluded. The other 67 patients with
SSIs (62.0\%) caused by identified microorganism(s) were enrolled in this retrospective study, and the culture-positive infection rate was $0.23 \%$. All the participants were provided informed consent before enrollment. This study was approved by our Institutional Review Board and was supported by two governmental grants (see the "Acknowledgments").

\section{Diagnostic criteria}

All the patients were evaluated in accordance with the guidelines for the prevention of SSI as defined by the Centers for Disease Control and Prevention, including the criteria for both superficial and deep SSIs [10]. Superficial SSIs included infections that occurred within 30 days after the operative procedure and involved only the skin and subcutaneous tissue of the incision. Deep SSIs included infections that appeared to be associated with the operative procedure and involved deep soft tissues of the incision (e.g., fasciae and muscle layers). Deep SSIs occurred within 30 days after the operative procedure if no implant was left in place or within 1 year if the implant remained in place. SSIs after spinal surgery can have an early onset and delayed appearance. We defined late infections as those that occurred $>30$ days after the primary surgery, whereas the early-onset infection occurred $\leq 30$ days postoperatively [11].

\section{Infection management}

All 67 patients with SSIs were administered antibiotics. Once SSIs were suspected, secretion from the incision or blood was sampled for bacterial culture and drug susceptibility tests before administering intravenous broad-spectrum antibiotics (second- or third-generation cephalosporins). Antibiotics were adjusted according to the outcome of the bacterial culture and drug susceptibility test, and oral antibiotics were administered if necessary. Two or more types of antibiotics were intravenously administered in patients with polymicrobial infection, whereas only one type of antibiotics was used in most patients with monomicrobial infection. To allow for continual adjustment of the dosage of antibiotics, an incision secretion sample was sent for bacterial culture and drug susceptibility tests every 2-3 days. Drug susceptibility tests showed that one patient with methicillin-resistant Staphylococcus aureus (MRSA) infection was sensitive to vancomycin at the be- 
ginning of antibiotic therapy, but turned to vancomycinintermediary staphylococcus aureus during the late period of treatment. The other patients did not develop resistance during the treatment period. During the course of the treatment, there was no emergence of new microorganism species. The number of microorganism species decreased in most polymicrobial infections, and some polymicrobial infections were converted to monomicrobial infection during the later periods of treatment.

After antibiotic therapy, clinical symptoms and signs (e.g., body temperature and wound) improved in seven of the 67 patients. Debridement was performed in other 60 patients, in whom little symptomatic improvement was achieved after 2-3 days of antibiotic treatment. In debridement, necrotic tissues and loosened grafted bones were removed, and the wound was repeatedly irrigated with hydrogen peroxide, diluted iodophor, and normal saline. Irrigation and drainage tubes were inserted into the wound before closure. After debridement, irrigation with sensitive antibiotics and drainage continued. The irrigation volume and time to remove the irrigation and drainage tubes depended on the result of the drainage culture. Sixty patients underwent debridement for an average of 1.5 times, with 18 of 60 patients undergoing debridement more than once. Implants were removed to control the infection in nine patients, including three patients with MRSA infection.

Use of antibiotics continued during the periprocedural period of debridement. Standard medicaltherapy following debridement included 6 weeks of intravenous antibiotics and, in some cases, long-term suppressive oral antibiotics if the surgeon and the infectious disease consultant thought this was indicated. Indications for withdrawal of antibiotics were as follows: normal body temperature; three consecutive negative bacterial cultures; and three consecutive normal white blood cell count and C-reactive protein level, with downtrend of erythrocyte sedimentation rate. After administration of antibiotics, hepatic and renal functions were monitored weekly. No side effects or abnormal hepatic and renal functions were noted during the administration of antibiotics.

\section{Data collection and grouping}

Data were compiled from electronic medical records. Detailed microbiological characteristics were gathered from the bacterial culture reports. Patients with polymicrobial infections had $\geq 2$ distinct cultures growing different species. In some patients, multiple organisms were cultured from the same surgical specimen or patient and each unique organism was counted as a positive culture. If the same organism was recovered from multiple specimens in an individual patient, it was counted once.

The patients were classified into either a monomicrobial infection group $(n=47)$ or a polymicrobial infection group $(n=20)$.

\section{Statistical analysis}

The $t$-test was used to compare the monomicrobial and polymicrobial infection groups with regard to age, body mass index (BMI), temperature, white blood cell count, percentage of neutrophils, hospitalization, number of rehospitalizations, number of debridements, duration of antibiotics administration, and number of antibiotics administered. The chi-squared test was used to compare the other categorical variables between the two groups. Statistical significance was considered as $P<0.05$. All the statistical analyses were performed using SPSS ver. 16.0 statistical software (SPSS, Chicago, IL, USA).

\section{Results}

\section{Homogeneity tests}

There were 47 patients in the monomicrobial group and 20 in the polymicrobial group. For the homogeneity test, age, gender distribution, and BMI were similar (Table 1).

Table 1. Homogeneity between the 2 groups

\begin{tabular}{lccc} 
Items & Monomicrobial group & Polymicrobial group & $p$-value \\
Age $(\mathrm{yr})$ & $50.74( \pm 14.99)$ & $51.05( \pm 15.55)$ & 0.940 \\
Male/Female & $28 / 19$ & $11 / 9$ & 0.728 \\
Body mass index $\left(\mathrm{kg} / \mathrm{m}^{2}\right)$ & $25.54( \pm 3.32)$ & $25.71( \pm 5.11)$ & 0.893 \\
\hline
\end{tabular}




\section{Microbiological tests}

In total, 108 patients were identified with SSIs after spinal surgery; in 67 patients (62.0\%), SSIs were culture-positive and caused by identified microorganism(s). Ninety-six strains of bacteria were identified from the bacterial culture of 67 SSIs (Table 2).

Among 96 strains, 60 (62.50\%) were gram-positive pathogenic bacteria, and the top three species were Staphylococcus aureus, Staphylococcus epidermidis, and Entero- coccus faecalis. Thirty-six strains (37.5\%) were gram-negative pathogenic bacteria, and the top three species were Escherichia coli, Enterobacter cloacae, and Acinetobacter baumannii. Antibiotic-resistant patients included three with methicillin-resistant Staphylococcus epidermidis and eight with methicillin-resistant Staphylococcus aureus.

Polymicrobial infections comprised 29.9\% (20/67) of SSIs after spinal surgery; 60\% (12/20) of polymicrobial infections contained both gram-positive and gram-negative bacteria, and $30 \%(6 / 20)$ of polymicrobial infections

Table 2. Pathogenic bacteria identified in 2 groups $^{\text {al }}$

\begin{tabular}{|c|c|c|}
\hline Bacteria & Strains (n) & Constituent ratio $(\%)$ \\
\hline Gram-positive & 60 & 62.50 \\
\hline Staphylococcus aureus & 33 & 34.38 \\
\hline Methicillin-sensitive Staphylococcus aureus & 25 & 26.04 \\
\hline Methicillin-resistant Staphylococcus aureus & 8 & 8.33 \\
\hline Staphylococcus epidermidis & 13 & 13.54 \\
\hline Methicillin sensitive Staphylococcus epidermidis & 10 & 10.42 \\
\hline Methicillin-resistant Staphylococcus epidermidis & 3 & 3.13 \\
\hline Enterococcus faecalis & 3 & 3.13 \\
\hline Staphylococcus capitis & 2 & 2.08 \\
\hline Staphylococcus warneri & 2 & 2.08 \\
\hline Corynebacterium & 2 & 2.08 \\
\hline Staphylococcus lentus & 1 & 1.04 \\
\hline Corynebacterium pseudodiphtheriticum & 1 & 1.04 \\
\hline Kocuria kristinae & 1 & 1.04 \\
\hline Streptococcus viridians & 1 & 1.04 \\
\hline Streptococcus pyogenes & 1 & 1.04 \\
\hline Gram-negative & 36 & 37.50 \\
\hline Escherichia coli & 10 & 10.42 \\
\hline Enterobacter cloacae & 5 & 5.21 \\
\hline Acinetobacter baumannii & 4 & 4.17 \\
\hline Klebsiella pneumonia & 3 & 3.13 \\
\hline Pseudomonas putida & 3 & 3.13 \\
\hline Pseudomonas aeruginosa & 3 & 3.13 \\
\hline Klebsiella oxytoca & 2 & 2.08 \\
\hline Stenotrophomonas maltophilia & 2 & 2.08 \\
\hline Serratia marcescens & 1 & 1.04 \\
\hline Comamonas acidovorans & 1 & 1.04 \\
\hline Comamonas testosteroni & 1 & 1.04 \\
\hline Citrobacter koseri & 1 & 1.04 \\
\hline Total & 96 & 100.00 \\
\hline
\end{tabular}

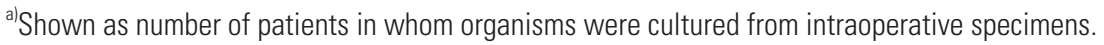


contained only gram-positive bacteria, whereas $10 \%(2 / 20)$ contained only gram-negative bacteria. Polymicrobial infections in $14(70 \%)$ patients were caused by two species of bacteria, infections in three patients were caused by three species of bacteria, infections in two patients were caused by four species of bacteria, and infection in one patient was caused by five species of bacteria. Bacterial strains in polymicrobial infections are listed in Table 3 , and the antibiotics administered to the patients in the polymicrobial group are shown in Table 4 .

\section{Clinical manifestation and management}

The majority of SSIs (64/67) occurred within 30 days of the initial surgery. The two groups were similar with regard to the following: surgical site, postoperative time to infection, infection site, incisional drainage, incisional swelling, incisional pain, neurological signs, temperature,

Table 3. Pathogenic bacteria in polymicrobial group $(n=20)$

\begin{tabular}{|c|c|c|}
\hline \multirow{2}{*}{ Case } & \multicolumn{2}{|c|}{ Bacteria } \\
\hline & Gram-positive & Gram-negative \\
\hline 1 & Staphylococcus warneri & $\begin{array}{l}\text { Escherichia coli } \\
\text { Stenotrophomonas maltophilia }\end{array}$ \\
\hline 2 & Methicillin-sensitive Staphylococcus aureus & Acinetobacter baumannii \\
\hline 3 & $\begin{array}{l}\text { Methicillin sensitive Staphylococcus epidermidis } \\
\text { Enterococcus faecalis }\end{array}$ & Comamonas acidovorans Comamonas testosteroni \\
\hline 4 & $\begin{array}{l}\text { Methicillin-resistant Staphylococcus epidermidis } \\
\text { Methicillin-sensitive Staphylococcus aureus }\end{array}$ & None \\
\hline 5 & $\begin{array}{l}\text { Methicillin-sensitive Staphylococcus aureus } \\
\text { Staphylococcus warneri }\end{array}$ & None \\
\hline 6 & Methicillin-resistant Staphylococcus epidermidis & Pseudomonas putida \\
\hline 7 & $\begin{array}{l}\text { Methicillin-resistant Staphylococcus aureus } \\
\text { Staphylococcus lentus }\end{array}$ & None \\
\hline 8 & None & $\begin{array}{l}\text { Pseudomonas aeruginosa } \\
\text { Pseudomonas putida }\end{array}$ \\
\hline 9 & $\begin{array}{l}\text { Methicillin-sensitive Staphylococcus aureus } \\
\text { Methicillin sensitive Staphylococcus epidermidis }\end{array}$ & None \\
\hline 10 & Methicillin-sensitive Staphylococcus aureus & Stenotrophomonas maltophilia \\
\hline 11 & $\begin{array}{l}\text { Corynebacterium pseudodiphtheriticum } \\
\text { Kocuria kristinae }\end{array}$ & None \\
\hline 12 & $\begin{array}{l}\text { Methicillin-resistant Staphylococcus aureus } \\
\text { Methicillin sensitive Staphylococcus epidermidis }\end{array}$ & $\begin{array}{l}\text { Acinetobacter baumannii } \\
\text { Escherichia coli }\end{array}$ \\
\hline 13 & None & $\begin{array}{l}\text { Klebsiella oxytoca } \\
\text { Enterobacter cloacae }\end{array}$ \\
\hline 14 & $\begin{array}{l}\text { Methicillin sensitive Staphylococcus epidermidis } \\
\text { Streptococcus viridians }\end{array}$ & Escherichia coli \\
\hline 15 & $\begin{array}{l}\text { Methicillin-resistant Staphylococcus aureus } \\
\text { Enterococcus faecalis }\end{array}$ & $\begin{array}{l}\text { Acinetobacter baumannii } \\
\text { Klebsiella pneumonia } \\
\text { Pseudomonas aeruginosa }\end{array}$ \\
\hline 16 & $\begin{array}{l}\text { Streptococcus pyogenes } \\
\text { Methicillin-sensitive Staphylococcus aureus }\end{array}$ & None \\
\hline 17 & Corynebacterium & $\begin{array}{l}\text { Pseudomonas aeruginosa } \\
\text { Pseudomonas putida }\end{array}$ \\
\hline 18 & Methicillin sensitive Staphylococcus epidermidis & Klebsiella pneumonia \\
\hline 19 & Methicillin-resistant Staphylococcus aureus & Escherichia coli \\
\hline 20 & Methicillin-resistant Staphylococcus aureus & Enterobacter cloacae \\
\hline
\end{tabular}


white blood cell counts, and percentage of neutrophils (Table 5).

To treat SSIs, debridement, irrigation-suction, or even implant removal was performed when necessary. The case

Table 4. Antibiotics used in polymicrobial group $(n=20)$

\begin{tabular}{lc} 
Antibiotics & Cases \\
Vancomycin & 10 \\
\hline Ciprofloxacin & 8 \\
\hline Ceftazidime & 5 \\
\hline Levofloxacin & 4 \\
\hline Cefuroxime & 4 \\
\hline Ceftriaxone & 3 \\
\hline Etimicin & 3 \\
\hline Piperacillin-sulbactam & 3 \\
\hline Imipenem-cilastin & 2 \\
\hline Meropenem & 2 \\
\hline Cefperazone-sulbactam & 2 \\
\hline Moxifloxacin & 2 \\
\hline Sulperazone & 1 \\
\hline Ampicillin & 1 \\
\hline Cefixime & 1 \\
\hline Clarithromycine & 1 \\
\hline Rifampin & 1 \\
\hline Clindamycin & 1 \\
\hline Metronidazole & 1 \\
\hline Cefotaxime & 1 \\
\hline Linezolid & 1 \\
\hline Cefepime & 1 \\
\hline & 1 \\
\hline
\end{tabular}

of a patient with wound infection with methicillin-resistant Staphylococcus aureus after lumbar surgery is shown in Fig. 1. The two groups were comparable in terms of hospital stays, number of rehospitalizations, number of debridements, duration of antibiotics administration, and number of antibiotics administered (Table 6). Among 47 patients in the monomicrobial group and 20 patients in the polymicrobial group, 45 and all patients underwent a spinal implant operation, respectively. Six of 45 patients in the monomicrobial infection group and three of 20 patients in the polymicrobial infection group required removal of the implant to control the infection. There were no significant differences in the implant removal rate between the two groups (Table 6).

\section{Treatment outcomes}

Amongthe 67 patients, one patient died of MRSA septicemia, which manifested as fever, alalia, and incisional swelling when the infection occurred. The patient had pulmonary and urinary tract infections during the period of hospitalization and ultimately died of multiple organ failure.

Sixty-six patients had healed wounds. Among 65 patients with internal fixation, the implants were maintained in 56 patients, whereas they were removed in nine patients. The infections in these nine patients were controlled by implant removal. All 66 patients were followed for 25177 months (average, 70 months), with no recurrence of infection at the last follow-up.

Table 5. Clinical manifestation of the 2 groups $^{\text {al }}$

\begin{tabular}{lccc} 
Clinical manifestation & Monomicrobial group & Polymicrobial group & $p$-value \\
Surgical site (cervical/thoracic/lumbar ) & $12 / 2 / 33$ & $3 / 3 / 14$ & 0.239 \\
\hline Infection (early/delayed) & $44 / 3$ & $20 / 0$ & 0.248 \\
\hline Infection (superficial/deep) & $19 / 28$ & $4 / 16$ & 0.107 \\
\hline Incisional drainage (Y/N) & $39 / 8$ & $14 / 6$ & 0.232 \\
\hline Incisional swelling (Y/N) & $19 / 28$ & $5 / 15$ & 0.228 \\
\hline Incisional pain (Y/N) & $15 / 32$ & $3 / 17$ & 0.153 \\
\hline Neurological signs (Y/N) & $2 / 45$ & $1 / 19$ & 0.893 \\
\hline Temperature $\left({ }^{\circ} \mathrm{C}\right)$ & $37.99( \pm 1.05)$ & $38.03( \pm 1.23)$ & 0.910 \\
\hline White blood cells (10 $/ \mathrm{L})$ & $10.68( \pm 4.07)$ & $13.03( \pm 7.65)$ & 0.106 \\
\hline Neutrophils $(\%)$ & $78.97( \pm 10.42)$ & $77.83( \pm 18.59)$ & 0.749 \\
\hline
\end{tabular}

$\mathrm{Y}$, stand for Yes; N, stand for No.

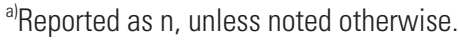



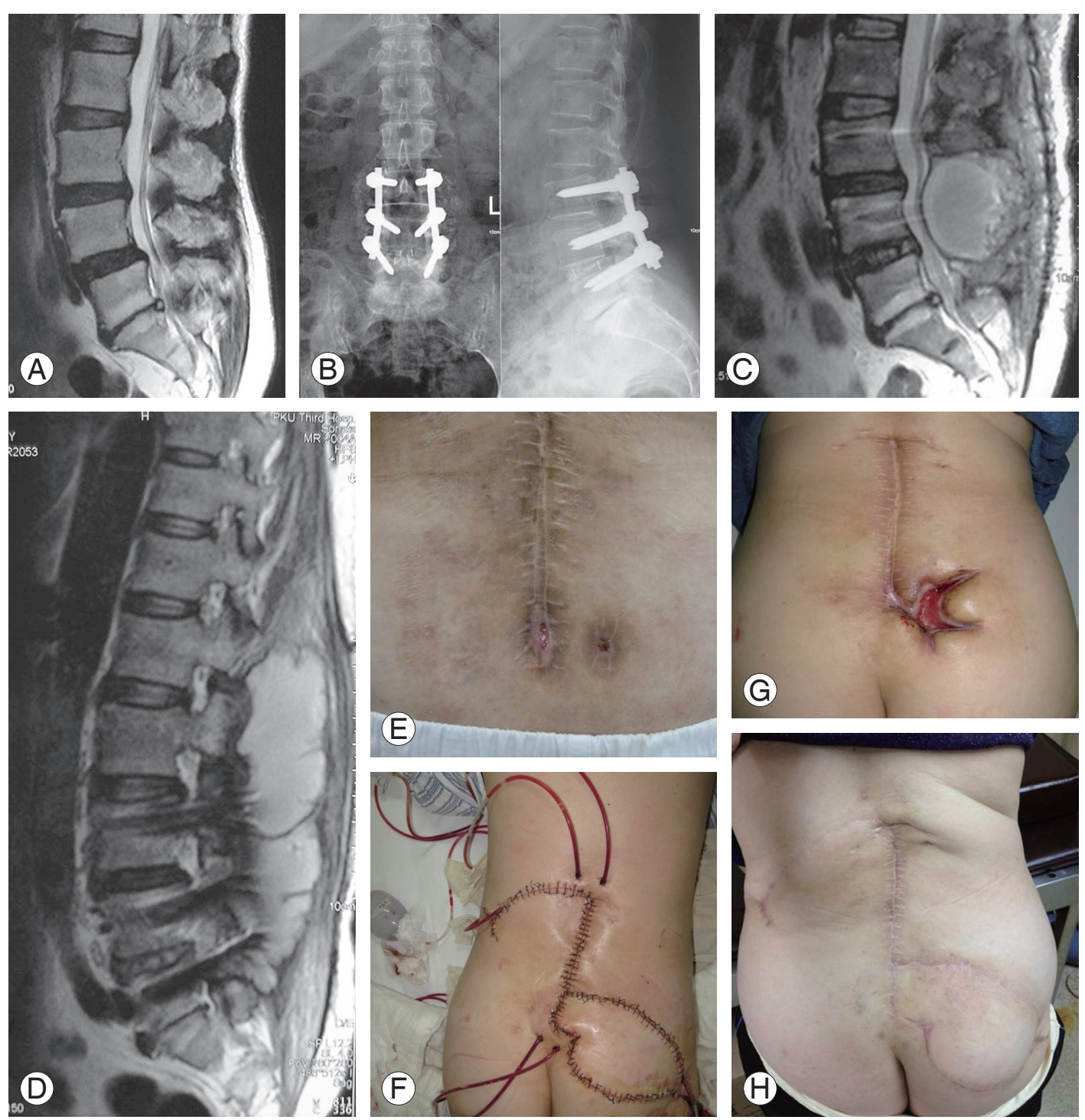

Fig. 1. A 59-year-old woman suffering from wound infection due to methicillin-resistant Staphylococcus aureus (MRSA) after lumbar surgery. The patient underwent five debridements. Implants were removed at the third debridement, and a gluteus maximus musculocutaneous flap was used to manage the complex wound at the fifth debridement. (A) Preoperative magnetic resonance imaging. (B) Postoperative roentgenographs (4 days postoperatively). (C) Wound seromas behind L3-5 (1 month postoperatively). (D) Wound abscess spread behind L1-S1; MRSA was found in the puncture fluid culture (12 months postoperatively). (E) A fistula was formed at the distal end of the incision, despite removal of implants (49 months postoperatively). (F) The fistula enlarged, although a fourth debridement was performed (61 months postoperatively). (G) A gluteus maximus musculocutaneous flap was used to manage the complex wound (62 months postoperatively). (H) The incision was in satisfactory condition without wound inflammation or secretion (70 months postoperatively).

Table 6. Management of the 2 groups

\begin{tabular}{lccc} 
Items & Monomicrobial group & Polymicrobial group & $p$-value \\
\hline Hospitalization (day) & $55.26 \pm 37.97$ & $69.00 \pm 47.96$ & 0.215 \\
\hline Rehospitalization (n) & $0.81 \pm 1.06$ & $0.65 \pm 1.39$ & 0.611 \\
\hline Debridement (n) & $1.32 \pm 1.02$ & $1.45 \pm 1.05$ & 0.636 \\
\hline Number of antibiotics (n) & $3.50 \pm 2.89$ & $3.43 \pm 1.89$ & 0.901 \\
\hline Duration of antibiotics (day) & $37.40 \pm 29.16$ & $42.55 \pm 27.83$ & 0.505 \\
\hline Implants removal (Y/N) & $6 / 39$ & $3 / 17$ & 0.857 \\
\hline
\end{tabular}

Y, stand for Yes; N, stand for No. 


\section{Discussion}

SSIs after spinal surgery are generally associated with serious morbidity, mortality, and increased resource utilization $[12,13]$. Antibiotics are commonly used to prevent and treat bacterial infections after spinal surgery [14]. However, the improper use of these medications may lead to even higher risk of infections as bacterial strains have the potential to evolve through the wide spread and frequent use of antibiotics. As a result, infections can become more difficult to control. Thus, monitoring the common infection-causing bacteria in a clinic is of considerable importance for guiding the rational use of antibiotics.

An unexpected finding of this study was the low culture-positive rate, which was $62.0 \%$. This is lower than the rate described in the literature, which ranges from $96.1 \%$ to $97.0 \%$ depending on the study populations $[15,16]$. The lower rate observed in our study may be because of the fact that antibiotics were administered in some patients before bacterial sampling, even when there was no secretion from the incision. Among 67 patients with SSIs, 14 (20.1\%) demonstrated no incisional drainage in this study (Table 5).

As defined earlier, polymicrobial infection refers to isolation of two or more species of microorganisms from at least two cultures of an incision secretion or intraoperative tissue specimen or isolation of two or more species of microorganisms in at least one intraoperative tissue specimen with evidence of infection in the surgical site. Bacterial contamination should be considered when a large number of colonies grow in noninoculated areas or more than three species of microorganisms are isolated in apetri dish with one sample. The clinical manifestation of polymicrobial infections differs from that of monomicrobial infections, especially with regard to drug resistance and therapeutic effects. In the present study, we investigated the influence of polymicrobial infections on clinical manifestation and severity. Unlike the report by Abdul-Jabbar et al. [15], we did not find any significant differences between the monomicrobial and polymicrobial groups regarding clinical manifestation or severity. This may be attributed to the different surgical procedures. In their study, surgical procedures involving the sacrum were significantly associated with polymicrobial infections. However, no sacrum surgical procedures were included in our study. Nevertheless, our data suggest no correlation between polymicrobial infections and surgical site (Table 5).
While polymicrobial infections may lead to unsatisfactory treatment outcomes, a multivariate logistic regression analysis suggested that polymicrobial infections are not an independent risk factor associated with the treatment failure [16]. Similar results were reported in another study of prosthetic joint infections [8]. The outcomes of polymicrobial prosthetic joint infections were similar to those of monomicrobial infections, and no statistically significant differences were found in 2-year free-of-failure survival between the two groups. In the study by Mok et al. [9], multiple debridements were significantly associated with polymicrobial infections, whereas in our present study, no significant differences were noted. Such differences in findings could be due to sampling size, as the former study included a relatively smaller number of patients (seven with monomicrobial and nine with polymicrobial infections) compared to ours (47 with monomicrobial and 20 with polymicrobial infections). The larger sample size may generate more reliable results. Nevertheless, for future study, additional high-quality, multi-center, largesample randomized controlled trials are required.

In this study, gram-positive organisms comprised the majority of pathogens isolated from cultures, and the top three are Staphylococcus aureus, Staphylococcus epidermidis, and Enterococcus faecalis. These results are consistent with earlier findings [15]. Previous efforts have suggested that the majority of the pathogenic bacteria isolated from early infections after spinal surgery are gram-positive cocci, especially Staphylococcus aureus, Staphylococcus epidermidis, and $\beta$-hemolytic streptococci [17]. Similar to our findings, the most common bacteria among isolated cocci is Staphylococcus aureus, with a rate of $>30 \%[18,19]$. Common gram-negative pathogenic bacteria during early infections wereEscherichia coli, Pseudomonas putida, Klebsiella pneumoniae, Enterobacter cloacae, Bacteroides spp., and Proteus vulgaris [17]. In the present study, the most predominant gram-negative bacteria found were Escherichia coli, Enterobacter cloacae, and Acinetobacter baumannii.

Gram-negative organisms accounted for $37.5 \%$ of the infections, which is higher than the rate described in the literature, whereas gram-negative organisms accounted for $0 \%-14.2 \%$ [20-22]. However, similar to our study's findings, Abdul-Jabbar et al. [15] reported that $30 \%$ of infections were caused by gram-negative organisms. The high incidence of gram-negative organism infections may be because of a certain number of lumbar infections 
(70.1\%) and may reflect skin flora in that region. However, it is unclear whether these infections are primarily due to intraoperative inoculation or postoperative contamination with fecal or urinary flora. Considering our results and previous reports, we can conclude that the above gramnegative and gram-positive pathogenic bacteria are commonly present, to a certain degree, at the surgical site after spinal surgery. This also indicates that the pathogenic bacteria that cause infections after spinal surgery are diverse and complex. Thus, cases of infection should be closely observed to achieve proper diagnosis and treatment. As for the medical therapy for the treatment of polymicrobial infection, we chose the most sensitive antibiotics based upon the antimicrobial susceptibility pattern of bacteria isolated from the cultures. In addition, drug combinations should be considered. Vancomycin-based and ciprofloxacin-based combinations were used for the majority of cases in our study (Table 4).

Unlike early infection, delayed infection has obscure and atypical symptoms because of low virulence and slow progression. Bacterial pathogens associated with delayed infection after spinal surgery are typically low-virulence strains colonizing the skin, such as coagulase-negative staphylococci, Propionibacterium acnes, and diphtheroids [19]. In the present study, there were three patients with delayed infections, which were caused by Staphylococcus aureus (two patients) and Staphylococcus epidermidis (one patient). According to a previous report [19], these bacteria may be coagulase-negative strains, and further confirmation may be needed. Furthermore, the source of delayed infections may be intraoperative contamination by low-virulence bacteria, which come out of hibernation as a response to damage of the surrounding tissue and a decrease in local resistance.

\section{Conclusions}

Our study suggests that the clinical manifestation and severity of polymicrobial infections after spinal surgery does not differ from that of monomicrobial infections. Nonetheless, the reasonable choice of sensitive antibiotics and a combination of antibiotics should be closely observed for the treatment of polymicrobial infections. The successful treatment of SSIs after spinal surgery requires thorough understanding of diagnostic and management principles.

\section{Conflict of Interest}

No potential conflict of interest relevant to this article was reported.

\section{Acknowledgments}

We hereby thank and acknowledge the support of the Beijing Municipal Science \& Technology Commission (Grant No. Z141107002514025) and the Capital Health Research and Development of Special (Grant No.2014-3-4096) towards this study.

\section{References}

1. Fang A, Hu SS, Endres N, Bradford DS. Risk factors for infection after spinal surgery. Spine (Phila $\mathrm{Pa}$ 1976) 2005;30:1460-5.

2. Collins I, Wilson-MacDonald J, Chami G, et al. The diagnosis and management of infection following instrumented spinal fusion. Eur Spine J 2008;17:44550.

3. Pull ter Gunne AF, Cohen DB. Incidence, prevalence, and analysis of risk factors for surgical site infection following adult spinal surgery. Spine (Phila Pa 1976) 2009;34:1422-8.

4. Kurtz SM, Lau E, Ong KL, et al. Infection risk for primary and revision instrumented lumbar spine fusion in the Medicare population. J Neurosurg Spine 2012; 17:342-7.

5. Calderone RR, Garland DE, Capen DA, Oster H. Cost of medical care for postoperative spinal infections. Orthop Clin North Am 1996;27:171-82.

6. Quaile A. Infections associated with spinal implants. Int Orthop 2012;36:451-6.

7. Janz V, Wassilew GI, Kribus M, Trampuz A, Perka C. Improved identification of polymicrobial infection in total knee arthroplasty through sonicate fluid cultures. Arch Orthop Trauma Surg 2015;135:1453-7.

8. Marculescu CE, Cantey JR. Polymicrobial prosthetic joint infections: risk factors and outcome. Clin Orthop Relat Res 2008;466:1397-404.

9. Mok JM, Guillaume TJ, Talu U, et al. Clinical outcome of deep wound infection after instrumented posterior spinal fusion: a matched cohort analysis. Spine (Phila Pa 1976) 2009;34:578-83.

10. Mangram AJ, Horan TC, Pearson ML, Silver LC, 
Jarvis WR. Guideline for Prevention of Surgical Site Infection, 1999. Centers for Disease Control and Prevention (CDC) Hospital Infection Control Practices Advisory Committee. Am J Infect Control 1999;27:97-132.

11. Liu C, Bayer A, Cosgrove SE, et al. Clinical practice guidelines by the infectious diseases society of america for the treatment of methicillin-resistant Staphylococcus aureus infections in adults and children. Clin Infect Dis 2011;52:e18-55.

12. Olsen MA, Nepple JJ, Riew KD, et al. Risk factors for surgical site infection following orthopaedic spinal operations. J Bone Joint Surg Am 2008;90:62-9.

13. Rao SB, Vasquez G, Harrop J, et al. Risk factors for surgical site infections following spinal fusion procedures: a case-control study. Clin Infect Dis 2011;53:686-92.

14. Dede O, Bosch P, Bowles AJ, Ward WT, Roach JW. Clinical decision making in early wound drainage following posterior spine surgery in pediatric patients. Spine Deformity 2014;2:104-9.

15. Abdul-Jabbar A, Berven SH, Hu SS, et al. Surgical site infections in spine surgery: identification of microbiologic and surgical characteristics in 239 cases. Spine (Phila Pa 1976) 2013;38:E1425-31.

16. Maruo K, Berven SH. Outcome and treatment of postoperative spine surgical site infections: predictors of treatment success and failure. J Orthop Sci 2014; 19:398-404.

17. Chaudhary SB, Vives MJ, Basra SK, Reiter MF. Postoperative spinal wound infections and postprocedural diskitis. J Spinal Cord Med 2007;30:441-51.

18. Wimmer C, Gluch H, Franzreb M, Ogon M. Predisposing factors for infection in spine surgery: a survey of 850 spinal procedures. J Spinal Disord 1998;11:124-8.

19. Weinstein MA, McCabe JP, Cammisa FP Jr. Postoperative spinal wound infection: a review of 2,391 consecutive index procedures. J Spinal Disord 2000;13: 422-6.

20. Rihn JA, Lee JY, Ward WT. Infection after the surgical treatment of adolescent idiopathic scoliosis: evaluation of the diagnosis, treatment, and impact on clinical outcomes. Spine (Phila Pa 1976) 2008;33:28994.

21. Viola RW, King HA, Adler SM, Wilson CB. Delayed infection after elective spinal instrumentation and fusion: a retrospective analysis of eight cases. Spine (Phila Pa 1976) 1997;22:2444-50.

22. Horwitz NH, Curtin JA. Prophylactic antibiotics and wound infections following laminectomy for lumber disc herniation. J Neurosurg 1975;43:727-31. 